\title{
Reliability of independent kinetic variables and measures of inter-limb asymmetry associated with \\ bilateral drop-landing performance
}

Received $24^{\text {st }}$ August 2018 Accepted $14^{\text {th }}$ September 2018 www.ijpefs.com

\author{
Louis Howe ${ }^{\mathrm{a},{ }^{*}}$ Jamie North ${ }^{\mathrm{b}}$, Mark Waldron b,c, Theodoros Bampouras a
}

Abstract: The purpose of this investigation was to establish the within-session reliability for peak vertical ground reaction force (vGRF), time to peak vGRF, and loading rate, both unilaterally and bilaterally, during a droplanding task as well as the reliability of inter-limb asymmetry in peak vGRF. Twenty-two men (age $=22 \pm 4$ years; height $=180.4 \pm 6.1 \mathrm{~cm}$; mass $=77.9 \pm 14.0 \mathrm{~kg}$ ) and 17 women (age $=20.4 \pm 3.6$ years; height $=164.6 \pm 9.4 \mathrm{~cm}$; mass $=60.3 \pm 9.8 \mathrm{~kg}$ ) volunteered for a single testing session. Participants completed three countermovement jumps (CMJ) to establish maximum jump height before performing five bilateral drop-landings from 50\%, $100 \%$, and $150 \%$ of their maximum CMJ height. The bilateral drop-landing protocol was then repeated after a 10 min recovery. Systematic bias, intraclass correlation coefficient (ICC), coefficient of variation (CV\%) and minimal detectable change (MDC) values for each kinetic measurement was calculated for the left and right leg, as well as bilaterally. There was no systematic bias present between trials $(P>0.05)$. All kinetic measurements showed relative reliability, ranging from large to near perfect (ICC $=0.57-0.95$ ). Absolute reliability ranged considerably depending on the measure and drop-height, with peak vGRF and time to peak GRF showing the greatest reliability at higher drop heights $(\mathrm{CV} \%=6.6-9.7 \%)$. Loading rate for all drop heights demonstrated $\mathrm{CV} \%$ ranging 13.0-27.6\%. Furthermore, MDC values for inter-limb asymmetries in peak vGRF ranged between $14.5-16.2 \%$ for all drop heights. Overall, many of the kinetic measurements evaluated were sufficiently reliable to detect typical changes in bilateral drop-landing performance when greater drop heights were used.

Key Words: within-session reliability, kinetic variables, landings; inter-limb asymmetry

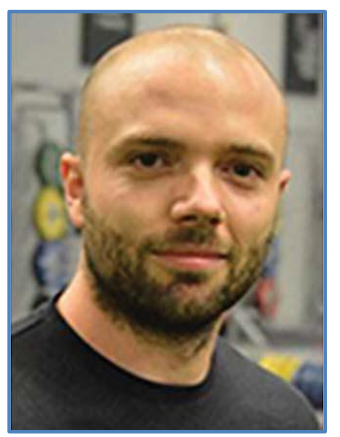

Louis Howe is a lecturer in Sports Rehabilitation at University of Cumbria.

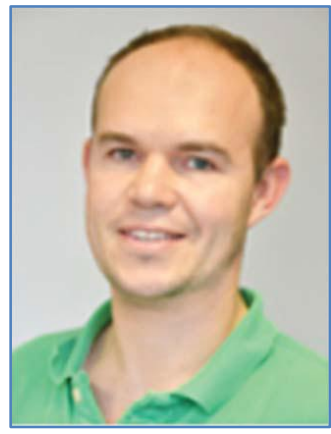

Jamie North is a reader in skill acquisition at St Mary's University, Twickenham. 


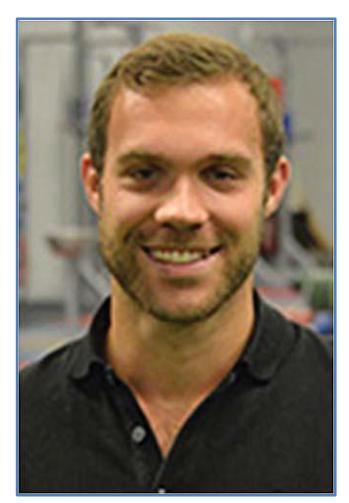

Mark Waldron is a senior lecturer in Exercise Physiology at St Mary's University, Twickenham

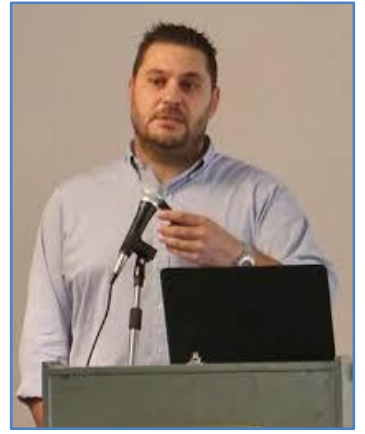

Dr Theodoros

M.

Bampouras holds a $\mathrm{PhD}$ from Manchester Metropolitan University on the implications of muscle mechanics on muscle activation capacity assessment and he is currently a Lecturer in Biomechanics at Lancaster University, UK. Theo's research interests lie primarily in the areas of muscle performance, mechanics and function. $\mathrm{He}$ has published work in muscle function assessment methods and muscle conditioning for improved power, as well as in the areas of balance, gait and vision in healthy older adults. Additionally, he has published work examining the validity and reliability of assessment methods and equipment

\section{Introduction}

Bilateral landings are commonly performed in court [1], team sports [2] and gymnastics [3]. When performing such tasks, peak vertical ground reaction forces (VGRF) can increase to multiples of bodyweight $[4,5]$. In order to attenuate such high forces, an athlete must adopt a coordinated movement strategy that flexes the ankle, knee and hip joints through the sagittal plane, such that the downward vertical rate of velocity of their centre of mass is progressively decelerated [6]. When coordination strategies to decelerate the centre of mass over a large range of motion are either not accessed as a movement solution [7], the result is a higher peak vGRF. Athletes who are exposed to greater peak vGRF during landings have an increased lower-extremity injury risk [1]. For example, Hewett et al. [8] showed that pre-screened female athletes who subsequently experienced anterior cruciate ligament injuries, produced normalized peak vGRF $20 \%$ higher than non-injured athletes during droplanding tasks. Additionally, athletes who display higher peak vGRF in the $100 \mathrm{~ms}$ following ground contact, place very high load on ligamentous structures located at the tibiofemoral joint [9].
As a result, the loading rate exhibited during landings may provide an insight into the stress being placed on various anatomical structures throughout the kinetic chain [10]. With bilateral drop-landings being commonly used to screen landing competency in athletic populations [11], reliability data that informs practice is required.

During bilateral landing activities, asymmetries in GRF are commonly identified [12, 13]. These asymmetries are an important consideration when working with athletes as they perform a high volume of bilateral landings as part of their physical preparation and competitive movements. Athletes who exhibit a large asymmetry in peak vGRF during bilateral landings may expose their dominant leg to excessive loading, thereby increasing the potential risk for overuse injury [14]. In such instances, reliable identification of bilateral asymmetry and subsequent interventions to reduce the magnitude of the asymmetry might be warranted and thus, in the first instance, it is necessary to investigate the reliability of asymmetries in kinetic variables during bilateral landings. 


\section{Louis Howe et al/2018}

Given that variables such as peak vGRF $(\mathrm{N})$, regularly performing a minimum of 30 minutes of

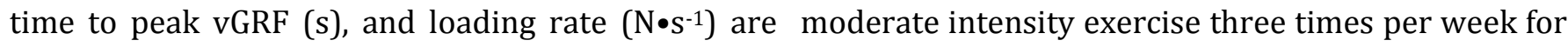
commonly reported in the literature as being at least six-months prior to testing [22]. Participants associated with injury risk $[8,10,15]$, coaches should were excluded if they had a history of lowerbe aware of the inherent error associated with extremity surgery or had lower-extremity injury sixtesting procedures. This includes error on behalf of months prior to testing. All participants were the athlete while performing a given protocol informed of the risks associated with the testing, (biological error) and that of the equipment prior to completing a pre-exercise questionnaire and (technical error) [16]. Although previous providing informed written consent. Ethical approval investigations have reported the reliability for was provided by the Institutional Research Ethics outcome measures relating to the propulsive phase Panel of the lead author.

of bilateral jumping tasks in various populations [1720], there is limited information on the kinetic factors associated with bilateral drop-landings [21], especially in regards to the presence of inter-limb asymmetries. The aim of this investigation was, therefore, to assess the reliability of peak vGRF, time to peak vGRF and loading rate, both bilaterally and unilaterally, during bilateral drop-landings from various landing heights, and to also establish the reliability for inter-limb asymmetries in peak vGRF during these landing tasks.

\section{Methods}

A within-session repeated measures design was used to establish the reliability for all kinetic variables related to bilateral drop-landings. Participants were required to report to the university laboratory for a single testing session. After familiarization, participants performed three countermovement jumps (CMJ) to establish maximum jump height for the landing task. Subsequently, participants performed five bilateral drop-landings from three heights: $50 \%$ of their maximum CMJ $100 \%$ of their maximum CMJ and $150 \%$ of their maximum CMJ. The participants then repeated the bilateral drop-landings from each height following a $10 \mathrm{~min}$ recovery.

\section{Participants}

Thirty-nine men ( $n=22$; age $=22 \pm 4$ years; height $=180.4 \pm 6.1 \mathrm{~cm}$; mass $=77.9 \pm 14.0 \mathrm{~kg}$ ) and women $(n=17$; age $=20.4 \pm 3.6$ years; height $=164.6$ $\pm 9.4 \mathrm{~cm}$; mass $=60.3 \pm 9.8 \mathrm{~kg}$ ) volunteered for this study. All reported to be physically active, defined as

\section{Procedures}

The participants performed a 5 minute standardized warm-up and three familiarization CMJ attempts. Countermovement jumps were performed from a standing position with each foot placed on a portable force platform recording at $1000 \mathrm{~Hz}$ (Pasco, Roseville, CA, USA). The force platforms were positioned side-by-side and embedded in custom built wooden mounts that were level with the force platforms, preventing any extraneous movement that could influence the force trace recorded. In bare feet, participants were informed to stand with their feet hip-width apart and with hands on their hips to eliminate the contribution of the arm swing. Participants were then asked to rapidly descend prior to explosively jumping as high as possible, with no control being placed on the depth or duration of the countermovement [23]. Upon landing, participants were required to ensure that full contact was made between each foot and the respective force platforms, with trials excluded if either foot made contact with the wooden mounts or neighbouring force platform. Following familarization, participants performed three CMJ for data analysis with a 60 second recovery between trials. Using a custommade Microsoft Excel spreadsheet, the force-time data was analysed using the time in the air method to calculate vertical jump height to the nearest $\mathrm{cm}$ [24]. The maximum value of the three attempts was then used to calculate box height for the bilateral droplandings.

Participants were given 10 minutes' recovery prior to repeating the standardized warm-up and 


\section{Louis Howe et al/2018}

performing three familiarization trials of bilateral both for each limb and bilaterally [28]. Time to peak drop-landings from each height. For the bilateral vGRF was then calculated as the time difference drop-landings, participants stood bare foot with their between initial contact and the time point where arms folded across their chest on a height-adjustable platform (to the nearest $1 \mathrm{~cm}$ ) positioned $15 \mathrm{~cm}$ away from the two force platforms. Participants then stepped off the height-adjustable platform, leading with the right leg, before immediately bringing the left leg off and alongside the right leg prior to ground contact. Participants were instructed to ensure they did not alter the vertical displacement of their centre of mass in this process so as to control for drop height [25]. Participants were asked to "land as softly as possible with both feet contacting the force platforms simultaneously and with equal weight distribution before returning to a standing position". This instruction was used in order to control for participants' focus of attention during the landing task between trials [26]. Full contact with the force platform was visually monitored throughout, with attempts disregarded if participants failed to either make full contact with the platform or maintain balance upon landing. No feedback was provided regarding the performance of the landing task. For data collection, participants performed five landings from drop heights of 50\%, $100 \%$ and $150 \%$ of their maximum CMJ height with a counterbalanced design employed to control for an order effect. Following each landing, 60 second recovery was provided before commencing the next trial. After a 10 minute recovery and standardized warm-up, participants repeated the bilateral drop-landing protocol, with drop height randomized for both trials 1 and 2 .

\section{Data analysis}

Raw vGRF data were low-pass filtered using a fourth-order Butterworth filter with a cut-off frequency of $50 \mathrm{~Hz}$ [27]. Peak vGRF, time to peak vGRF, and loading rate was then calculated unilaterally for the right and left leg, as well as bilaterally. For bilateral measures, both the left and right force data were summed prior to analysis. Peak vGRF data was normalized to body mass $\left(\mathrm{N} \bullet \mathrm{kg}^{-1}\right)$. For time to peak vGRF to be determined, initial contact was identified as the point that vGRF exceeded $10 \mathrm{~N}$ peak vGRF occurred. Loading rate was calculated as normalized peak vGRF divided by time to peak vGRF [29]. To calculate inter-limb asymmetries in peak vGRF, the asymmetry index equation was performed for each landing as outlined by Jordan et al. [30]:

Asymmetry Index $=($ Right peak vGRF - Left peak vGRF) *100 / (Right peak vGRF + Left peak vGRF)

where a positive value was arbitrarily assigned to right leg dominance, while a negative number indicated left leg dominance. All force-time measures were averaged across the five landings for each trial.

\section{Statistical analysis}

Descriptive statistics (means \pm standard deviation) were calculated for all variables. The assumption of normality was confirmed using the Shapiro-Wilk test. To examine for heteroscedastic errors, the relationship between the mean values between tests and the difference between repeat tests was evaluated using Pearson's coefficient. The within-session reliability for peak vGRF, time to peak vGRF, and loading rate for each limb (left and right) and bilaterally, along with asymmetries in peak vGRF between limbs, was initially assessed using a paired samples $t$-test to calculate systematic bias between trial 1 and 2 from each box height [16]. The $\alpha$-priori level of significance was set at $P<0.05$, with a Bonferroni correction applied post-hoc to the $\alpha$-level for the ten variables pairwise between-comparisons (i.e. $0.05 / 10=P=0.005$ ) from each box height in order to reduce the risk of type I errors [31]. Relative reliability was determined using an intra-class correlation coefficient (ICC) as suggested by Atkinson and Nevill [16] and reported with 95\% confidence intervals, with ICCs interpreted as follows: 0.01-0.3 poor, 0.3-0.5 moderate, 0.5-0.7 large, 0.7-0.9 very large, and >0.9 nearly perfect [32]. Absolute reliability was calculated using the coefficient of variation (CV\%), the 95\% limits of agreement, standard error of measurement (SEM; SEM = SD $\sqrt{1}$ ICC) [16] and minimal detectable change (MDC; MDC $=\mathrm{SEM}^{*} 1.96 * \sqrt{2}$ ) [33]. Due to the peak vGRF 
asymmetry being interval data, CV\% was not bilateral drop-landing performance have relative calculated for this variable. ICC and CV\% were reliability ranging from large to near perfect, with calculated using customised Microsoft Excel absolute reliability (represented by CV\%) ranging spreadsheet available online [34]. The CV\% was used from 6.6-27.6\%. Therefore, the bilateral dropas the primary measure of absolute reliability but we landing can be reliably used as a screening tool for have reported a variety of statistical interpretations athlete populations, although the variability in error to facilitate wider applications or different will be strongly influenced by the force-time preferences of researchers or practitioners. All measurement analysed and the magnitude of change statistical tests were performed using SPSS® being detected [16].

statistical software package (v.24; SPSS Inc., Chicago, IL, USA).

\section{Results}

The group mean for $\mathrm{CMJ}$ height was $29.8 \pm$ $8.1 \mathrm{~cm}$. Mean and standard deviations for all variables are presented in Tables 1-4. There was no systematic bias or heteroscedasticity found between trials 1 and 2 for any variable for each drop height. For measures of peak vGRF, relative reliability was nearly perfect (ICC $\geq 0.90$ ) for all variables except peak vGRF on the right extremity from the $50 \% \mathrm{CMJ}$ drop height, which had very large relative reliability (ICC $=0.87$ ). Measures of absolute reliability for peak vGRF are reported in Table 1, with CV\% ranging from $7.1-13.0 \%$ for all variables. Time to peak vGRF demonstrated relative reliability of large to near perfect across all drop heights (ICC $=0.57-0.92$ ). However, absolute reliability was greater for drop heights of $150 \% \mathrm{CMJ}$ height (CV\% $=6.6-9.5 \%)$ when compared to drop heights of $100 \% \mathrm{CMJ}$ height (CV\% $=10.5-13.1 \%)$ and 50\% CMJ height (CV\% = 14.9$27.6 \%$ ) for time to peak vGRF (Table 2). Loading rate possessed very large to near perfect relative reliability (ICC $=0.86-0.95$ ) across all drop heights, and absolute reliability establishing $\mathrm{CV} \%$ ranging between 13.0-27.6\% (Table 3). Measures of reliability for asymmetries in peak vGRF are shown in Table 4, with relative reliability shown to be very large $($ ICC $=0.72-0.74)$.

\section{Discussion}

The primary purpose of this study was to establish the within-session reliability for force-time measures of the bilateral drop-landing from drop heights of $50 \%, 100 \%$ and $150 \%$ of maximum CMJ height. Our data shows that kinetic measures of

Importantly, no systematic bias was detected between trials using the within-session design, indicating that no learning effect, participant bias, or acute adaptations were present between trials [16]. These findings suggest that the procedures used for this investigation were appropriate for diminishing the effects of systematic error. Practitioners, however, should remain aware of such considerations when designing procedures for testing an athlete's landing capabilities in order to reduce error and allow for better interpretation of their data [33].

Similar findings have previously been identified, with James et al. [21] reporting relative reliability as very large for bilateral measures of peak vGRF (ICC $=0.77$ ) and loading rate (ICC $=0.87$ ) for bilateral drop-landings from a $61 \mathrm{~cm}$ box. Similarly, using a within-session design, Walsh et al. [35] reported near perfect reliability for peak vGRF and time to peak vGRF (ICC $=0.98$ and 0.92 , respectively) following a bilateral drop-landing from a $31 \mathrm{~cm}$ box. Collectively, our findings support previous investigations; however, we have extended our interpretation of measurement error by quantifying absolute reliability (i.e. agreement) for all variables, across varying box heights for both unilateral and bilateral measures.

The ICC's for bilateral and unilateral measures of peak vGRF across each drop height ranged from $0.87-0.95$, with $\mathrm{CV} \%$ between 7.1$13.0 \%$ (Table 1). Although the ICC values suggested peak vGRF during bilateral landings to be arbitrarily reliable, it has been suggested that $<10 \%$ for $\mathrm{CV} \%$ is the acceptable threshold for a test measure to be deemed reliable [36]. 


\section{Louis Howe et al /2018}

Table 1 Within-session reliability for normalized peak vGRF for bilateral drop-landing from all drop height.

\begin{tabular}{|c|c|c|c|c|c|c|c|c|}
\hline & Trial 1 & Trial 2 & Change in mean & $95 \%$ LOA & ICC (95\% CI) & $\mathrm{CV}(\%)$ & SEM & MDC \\
\hline & Mean \pm SD & Mean \pm SD & & & & & & \\
\hline \multicolumn{9}{|c|}{ Drop height $50 \%$ of maximum CMJ height } \\
\hline Total peak vGRF $\left(\mathrm{N} \bullet \mathrm{kg}^{-1}\right)$ & $2.74 \pm 0.91$ & $2.71 \pm 0.91$ & -0.03 & $0.03 \pm 0.79$ & $0.90(0.84-0.94)$ & 9.4 & 0.28 & 0.78 \\
\hline Right peak vGRF $\left(\mathrm{N} \cdot \mathrm{kg}^{-1}\right)$ & $1.76 \pm 0.64$ & $1.70 \pm 0.54$ & -0.06 & $0.06 \pm 0.61$ & $0.87(0.78-0.92)$ & 13.0 & 0.21 & 0.60 \\
\hline Left peak vGRF $\left(\mathrm{N} \bullet \mathrm{kg}^{-1}\right)$ & $1.23 \pm 0.41$ & $1.22 \pm 0.44$ & 0.01 & $0.01 \pm 0.33$ & $0.92(0.87-0.96)$ & 10.0 & 0.12 & 0.32 \\
\hline \multicolumn{9}{|c|}{ Drop height $100 \%$ of maximum CMJ height } \\
\hline Total peak vGRF $\left(\mathrm{N} \cdot \mathrm{kg}^{-1}\right)$ & $3.41 \pm 1.17$ & $3.21 \pm 0.95$ & -0.20 & $0.20 \pm 0.85$ & $0.92(0.87-0.95)$ & 8.8 & 0.30 & 0.83 \\
\hline Right peak vGRF $\left(\mathrm{N} \bullet \mathrm{kg}^{-1}\right)$ & $2.02 \pm 0.75$ & $1.93 \pm 0.63$ & -0.10 & $0.10 \pm 0.56$ & $0.92(0.86-0.95)$ & 10.1 & 0.20 & 0.55 \\
\hline Left peak vGRF $\left(\mathrm{N} \cdot \mathrm{kg}^{-1}\right)$ & $1.62 \pm 0.58$ & $1.54 \pm 0.51$ & -0.09 & $0.09 \pm 0.46$ & $0.91(0.86-0.95)$ & 11.2 & 0.16 & 0.45 \\
\hline \multicolumn{9}{|c|}{ Drop height $150 \%$ of maximum CMJ height } \\
\hline Total peak vGRF $\left(\mathrm{N} \cdot \mathrm{kg}^{-1}\right)$ & $4.18 \pm 1.27$ & $3.99 \pm 1.28$ & -0.18 & $0.18 \pm 0.77$ & $0.95(0.92-0.97)$ & 7.1 & 0.27 & 0.75 \\
\hline Right peak vGRF $\left(\mathrm{N} \cdot \mathrm{kg}^{-1}\right)$ & $2.43 \pm 0.80$ & $2.32 \pm 0.78$ & -0.11 & $0.11 \pm 0.65$ & $0.92(0.86-0.95)$ & 9.6 & 0.23 & 0.63 \\
\hline Left peak vGRF $\left(\mathrm{N} \bullet \mathrm{kg}^{-1}\right)$ & $2.11 \pm 0.75$ & $2.06 \pm 0.76$ & -0.06 & $0.06 \pm 0.49$ & $0.95(0.91-0.97)$ & 9.7 & 0.17 & 0.47 \\
\hline
\end{tabular}

Notes: $\mathrm{vGRF}=$ Vertical ground reaction forces; $\mathrm{LOA}=$ Limits of agreement; ICC = Intraclass correlation coefficient; $\mathrm{CV}=\mathrm{Coefficient}$ of variation; CI = Confidence interval; SEM = Standard error of measurement; MDC = Minimal detectable change. ${ }^{*}=$ Significant difference between trial 1 and 2. 


\section{Louis Howe et al/2018}

Table 2. Within-session reliability for time to peak vGRF for bilateral drop-landing from all drop heights.

\begin{tabular}{|c|c|c|c|c|c|c|c|c|}
\hline & Trial 1 & Trial 2 & Change & $95 \% \mathrm{LOA}$ & ICC $(95 \% \mathrm{CI})$ & $\mathrm{CV}(\%)$ & SEM & MDC \\
\hline & Mean \pm SD & Mean \pm SD & in mean & & & & & \\
\hline \multicolumn{9}{|c|}{ Drop height $50 \%$ of maximum CMJ height } \\
\hline Total time to peak vGRF (s) & $0.088 \pm 0.031$ & $0.092 \pm 0.035$ & 0.004 & $-0.004 \pm 0.038$ & $0.84(0.74-0.90)$ & 15.9 & 0.013 & 0.037 \\
\hline Right time to peak vGRF (s) & $0.077 \pm 0.022$ & $0.081 \pm 0.025$ & 0.005 & $-0.005 \pm 0.033$ & $0.75(0.61-0.85)$ & 14.9 & 0.012 & 0.033 \\
\hline Left time to peak vGRF (s) & $0.114 \pm 0.057$ & $0.108 \pm 0.045$ & -0.006 & $0.006 \pm 0.094$ & $0.57(0.37-0.73)$ & 27.6 & 0.034 & 0.093 \\
\hline \multicolumn{9}{|c|}{ Drop height $100 \%$ of maximum CMJ height } \\
\hline Total time to peak vGRF (s) & $0.068 \pm 0.023$ & $0.068 \pm 0.022$ & 0.000 & $-0.004 \pm 0.034$ & $0.91(0.84-0.94)$ & 10.7 & 0.007 & 0.019 \\
\hline Right time to peak vGRF (s) & $0.065 \pm 0.021$ & $0.064 \pm 0.015$ & -0.001 & $0.001 \pm 0.021$ & $0.84(0.74-0.90)$ & 10.5 & 0.007 & 0.020 \\
\hline Left time to peak vGRF (s) & $0.080 \pm 0.035$ & $0.080 \pm 0.035$ & 0.000 & $0.000 \pm 0.033$ & $0.89(0.82-0.94)$ & 13.1 & 0.011 & 0.032 \\
\hline \multicolumn{9}{|c|}{ Drop height $150 \%$ of maximum CMJ height } \\
\hline Total time to peak vGRF (s) & $0.055 \pm 0.014$ & $0.056 \pm 0.014$ & 0.001 & $-0.001 \pm 0.017$ & $0.82(0.72-0.89)$ & 9.5 & 0.006 & 0.016 \\
\hline Right time to peak vGRF (s) & $0.053 \pm 0.012$ & $0.054 \pm 0.012$ & 0.001 & $-0.001 \pm 0.010$ & $0.91(0.85-0.95)$ & 6.6 & 0.004 & 0.010 \\
\hline Left time to peak vGRF (s) & $0.063 \pm 0.027$ & $0.063 \pm 0.023$ & 0.000 & $0.000 \pm 0.021$ & $0.92(0.86-0.95)$ & 8.7 & 0.007 & 0.020 \\
\hline
\end{tabular}

Notes: $\mathrm{vGRF}=$ Vertical ground reaction forces; $\mathrm{LOA}=$ Limits of agreement; $\mathrm{ICC}=$ Intraclass correlation coefficient; $\mathrm{CV}=\mathrm{Coefficient}$ of variation; $\mathrm{CI}=$ Confidence interval; SEM = Standard error of measurement; MDC = Minimal detectable change. ${ }^{*}=$ Significant difference between trial 1 and 2. 


\section{Louis Howe et al/2018}

Table 3. Within-session reliability for loading rate for bilateral drop-landing from all drop heights.

\begin{tabular}{|c|c|c|c|c|c|c|c|c|}
\hline & Trial 1 & Trial 2 & Change in mean & $95 \% \mathrm{LOA}$ & ICC $(95 \% \mathrm{CI})$ & $\mathrm{CV}(\%)$ & SEM & MDC \\
\hline & Mean \pm SD & Mean \pm SD & & & & & & \\
\hline \multicolumn{9}{|c|}{ Drop height $50 \%$ of maximum CMJ height } \\
\hline Total loading rate $(\mathrm{N} / \mathrm{s})$ & $40.3 \pm 25.3$ & $38.7 \pm 27.9$ & -1.6 & $1.60 \pm 26.33$ & $0.88(0.80-0.93)$ & 20.9 & 9.3 & 25.7 \\
\hline Right loading rate $(\mathrm{N} / \mathrm{s})$ & $28.1 \pm 18.0$ & $25.8 \pm 16.2$ & -2.3 & $2.30 \pm 16.80$ & $0.88(0.80-0.93)$ & 23.4 & 5.9 & 16.4 \\
\hline Left loading rate $(\mathrm{N} / \mathrm{s})$ & $16.2 \pm 11.6$ & $16.2 \pm 13.7$ & 0.0 & $0.02 \pm 13.44$ & $0.86(0.77-0.92)$ & 27.6 & 4.7 & 13.2 \\
\hline \multicolumn{9}{|c|}{ Drop height $100 \%$ of maximum CMJ height } \\
\hline Total loading rate $(\mathrm{N} / \mathrm{s})$ & $61.5 \pm 37.9$ & $54.8 \pm 27.3$ & -6.7 & $6.70 \pm 30.91$ & $0.89(0.82-0.94)$ & 16.1 & 10.9 & 30.2 \\
\hline Right loading rate $(\mathrm{N} / \mathrm{s})$ & $38.0 \pm 24.0$ & $35.0 \pm 19.3$ & -3.0 & $3.03 \pm 17.26$ & $0.92(0.87-0.95)$ & 16.7 & 6.1 & 16.8 \\
\hline Left loading rate $(\mathrm{N} / \mathrm{s})$ & $27.1 \pm 18.9$ & $24.0 \pm 14.0$ & -3.1 & $3.08 \pm 15.55$ & $0.89(0.82-0.94)$ & 22.8 & 5.5 & 15.2 \\
\hline \multicolumn{9}{|c|}{ Drop height $150 \%$ of maximum CMJ height } \\
\hline Total loading rate $(\mathrm{N} / \mathrm{s})$ & $86.6 \pm 42.5$ & $81.1 \pm 41.7$ & -5.5 & $5.47 \pm 26.70$ & $0.95(0.92-0.97)$ & 13.0 & 9.4 & 26.0 \\
\hline Right loading rate $(\mathrm{N} / \mathrm{s})$ & $52.0 \pm 27.4$ & $49.3 \pm 27.4$ & -2.7 & $2.74 \pm 19.14$ & $0.94(0.90-0.96)$ & 14.0 & 6.7 & 18.7 \\
\hline Left loading rate $(\mathrm{N} / \mathrm{s})$ & $41.3 \pm 24.1$ & $40.1 \pm 24.5$ & -1.3 & $1.27 \pm 15.05$ & $0.95(0.92-0.97)$ & 17.0 & 5.3 & 14.7 \\
\hline
\end{tabular}

Notes: $\mathrm{LOA}=$ Limits of agreement; ICC = Intraclass correlation coefficient; CV = Coefficient of variation; CI = Confidence interval; $\mathrm{SEM}=\mathrm{Standard}$ error of measurement; MDC = Minimal detectable change. ${ }^{*}=$ Significant difference between trial 1 and 2. 


\section{Louis Howe et al /2018}

Table 4. Within-session reliability for peak vGRF asymmetry for bilateral drop-landing from all drop heights.

\begin{tabular}{|c|c|c|c|c|c|c|c|}
\hline & Trial 1 & Trial 2 & Change in mean & $95 \% \mathrm{LOA}$ & $\mathrm{ICC}(95 \% \mathrm{CI})$ & SEM & MDC \\
\hline & Mean \pm SD & Mean \pm SD & & & & & \\
\hline Peak vGRF asymmetry at 50\% CMJ (\%) & $17.4 \pm 10.6$ & $16.5 \pm 11.6$ & -0.9 & $0.89 \pm 16.50$ & $0.72(0.57-0.83)$ & 5.9 & 16.2 \\
\hline Peak vGRF asymmetry at $100 \% \mathrm{CMJ}(\%)$ & $10.9 \pm 9.8$ & $11.3 \pm 10.9$ & 0.4 & $-0.41 \pm 14.82$ & $0.74(0.60-0.84)$ & 5.3 & 14.6 \\
\hline Peak vGRF asymmetry at $150 \% \mathrm{CMJ}(\%)$ & $7.7 \pm 9.8$ & $6.7 \pm 10.8$ & -0.9 & $0.91 \pm 15.28$ & $0.73(0.57-0.83)$ & 5.4 & 15.0 \\
\hline
\end{tabular}

Notes $: \mathrm{CMJ}=$ Countermovement jump height; $\mathrm{vGRF}=$ Vertical ground reaction forces; $\mathrm{LOA}=$ Limits of agreement; ICC $=$ Intraclass correlation coefficient; $\mathrm{CI}=$ Confidence interval; $\mathrm{SEM}=$ Standard error of measurement; $\mathrm{MDC}=$ Minimal detectable change. $*=$ Significant difference between trial 1 and 2 . 


\section{Louis Howe et al /2018}

This practice for determining absolute reliability previously shown to differ by approximately $12.3 \%$ would indicate that unilateral measures of peak VGRF bilaterally between gymnasts and recreational during the bilateral drop-landing from heights of athletes from a drop landing of $30 \mathrm{~cm}$ [39]. If this $50 \%$ and $100 \%$ of an individual's CMJ height should be considered to lack the necessary reliability (Table 1). Similarly, time to peak vGRF CV\% ranged from $10.5-27.6 \%$ for bilateral drop-landings at $50 \%$ and $100 \%$ of $\mathrm{CMJ}$ height, both bilaterally and unilaterally (Table 2), resulting in the same arbitrary outcome of unacceptable reliability. However, the use of this arbitrary cut-off point has been contested on the basis that that it is not based on a well-defined analytical goal [16]. Therefore, as part of our investigation, we purposely chose not to apply an arbitrary $10 \%$ threshold for CV\% to determine reliability. Instead, practitioners should appreciate that measurements of peak vGRF and time to peak vGRF during bilateral drop-landings, are likely to be more variable at lower drop heights and evaluate this in conjunction with the anticipated or likely signal changes. For example, $\mathrm{Vu}$ et al. [37] previously showed that firefighters performing bilateral droplandings from a $41 \mathrm{~cm}$ drop height wearing restrictive firefighting boots were exposed to $10.8 \%$ greater peak vGRF bilaterally, when compared to landings in athletic footwear. Based on our data, the increase in peak vGRF associated with wearing firefighting boots would be defined as real from any drop-height between the individuals' $50-150 \%$ CMJ height. However, in a study by Milner et al. [26] investigating the effects of verbal instruction on a bilateral landing task, an instructional cue to land with knees over your toes led to a 9.0\% mean reduction in bilateral peak vGRF across their cohort. Had this landing been performed from a drop height equalling $50 \%$ of each individual's maximum CMJ height, this reduction in peak vGRF would reside within the boundaries of measurement error and could not be defined as real change. As changes in landing mechanics have been shown to invoke an increase in peak vGRF of up to $29.6 \%$ bilaterally [38], we suggest that $\mathrm{CV} \%$ reported in our investigation for peak vGRF may still be low enough to identify changes in an athlete's capacity to successfully attenuate forces across all drop heights. Similarly, differences in time to peak vGRF have been drop height equated to the participants $100 \% \mathrm{CMJ}$ height, this difference in time to peak vGRF would exceed the CV\% of $10.7 \%$ established in our investigation, and therefore present as a meaningful difference between cohorts. Therefore, we recommend that practitioners appreciate the measurement error established in our investigation for kinetic measures associated with bilateral landings to interpret an athlete's competency to dissipate forces. This interpretation must be made relative to the athlete's maximum CMJ height, as lower drop heights produce greater variability in measurement error.

Loading rate has previously been suggested to be an important mechanical variable to consider during landing activities, as it relates to injury risk [40]. The mean loading rates increased proportionally with box height. However, the CV\% for loading rate observed was among the largest, particularly at lower drop-heights. Yet, loading rate measured bilaterally during drop-landings from 61 $\mathrm{cm}$, have been shown to acutely decrease by $23 \%$ following a fatigue protocol [41]. Furthermore, significant reductions in ankle plantar flexion angles at initial contact have been shown to increase loading rate bilaterally by $711 \%$, rising from $47.99 \mathrm{~N} / \mathrm{s}$ to $341.16 \mathrm{~N} / \mathrm{s}$ [13]. When compared to our data, such changes would be regarded as meaningful across all drop heights relative to the CV\% reported in Table 3. With such large changes acutely observed, it is likely that differences in loading rate can be detected, although the magnitude of change will need to be relatively large depending on drop height.

The change reported herein between box height and the reliability of landing kinetics supports the findings of recent investigations [42], where the variability (CV\%) in lower-limb joint moments were reduced as a function of drop height, which ranged from $20 \%$ to $180 \%$ of CMJ height. It was suggested that the reduced variability in joint moments observed with increased landing heights indicated a more consistent, yet potentially harmful, reliance on 


\section{Louis Howe et al/2018}

selected joint structures during more demanding tasks, which may increase injury risk [42]. Here, we expand upon these findings by reporting the reduced variability of kinetic drop-landing profiles at greater box heights. More specifically, our data indicate that the relative variability for peak VGRF, time to peak vGRF, and loading rate measured both bilaterally and unilaterally, all decreased with greater drop heights.

For practical purposes, we established the MDC values for all force-time variables. These values allow for practitioners to identify whether an intervention has resulted in 'meaningful' change [33]. An example of this could be a reduction in the peak vGRF an individual is exposed to during bilateral droplandings. An athlete performing a bilateral droplanding from a drop height of 50\% CMJ height with the bilateral peak vGRF of $2.5 \mathrm{~N} \bullet \mathrm{kg}^{-1}$, would need to reduce peak VGRF by $>0.78 \mathrm{~N} \bullet \mathrm{kg}^{-1}$ for the change to be defined as meaningful. Likewise, if the same athlete were to present with bilateral peak vGRF of $4.8 \mathrm{~N} \cdot \mathrm{kg}^{-1}$ from a drop height of $150 \%$ CMJ height, a reduction of $>0.75 \mathrm{~N} \cdot \mathrm{kg}^{-1}$, would be required for the intervention to be deemed successful. These MDC values represent changes in peak vGRF of $31 \%$ and $16 \%$ from drop heights equating to $50 \%$ and $150 \%$ of CMJ height, respectively. This example further illustrates the need to identify drop heights for screening landing mechanics relative to the athletes CMJ height when interpreting force-time data. However, practitioners should be aware that the use of MDC values to define a change as meaningful for an individual remains somewhat arbitrary and is based on a number of assumptions, such as data being distributed normally [16]. It may be that analytical goals for identifying real change following an intervention be based on practical outcomes that are driven by the literature relevant to the kinetic measurement being assessed relative to the demographic profile of the population.

Asymmetries during athletic activities have been suggested to impair performance outcomes [43] and increase injury risk [14, 44]. Our investigation showed that a large amount of variability in peak vGRF asymmetry existed during the bilateral drop-landings, with MDC values larger than, or approaching, the mean asymmetry observed in our population across all drop heights (Table 4). This is similar to previous findings [14], with the asymmetries in vGRF during bilateral landings appearing to vary greatly between trials. Inter-limb asymmetries in force profiles during bilateral landings are particularly important metrics among post-rehabilitation athletes. For example, Paterno et al. [29] found that a group of female athletes, who had returned to sport two years after anterior cruciate ligament reconstructive surgery, demonstrated side-to-side vGRF asymmetries during a drop vertical jump. These asymmetries were in favour of the uninvolved limb and resulted in a mean difference of $0.5 \mathrm{x}$ bodyweight in peak vGRF, representing a mean asymmetry index score of $14.3 \%$ [29]. If this magnitude of asymmetry was found during the performance of a bilateral droplanding task, based on the MDC values presented in Table 4, this asymmetry value would not present as meaningful, regardless of drop height. Therefore when screening for asymmetries during bilateral drop-landings, our investigation suggests that peak vGRF should be analyzed with caution due to the error associated with this outcome variable. Although a number of possibilities exist for why such high levels of variability in asymmetry for peak vGRF were present, the training background of the participants included in our investigation may have prompted the high level of variability observed between trials. Recently it has been shown that athletes who are highly familiar with performing specific landing tasks exhibit less variability in interlimb asymmetries relative to novice athletes [46]. Novice athletes who are less familiar with landing tasks may demonstrate greater inter-limb variability in their movement strategies between trials while they explore adaptive behaviours in search of coordination solutions to the movement problem [45]. Therefore, our findings may not be applicable to individuals well-trained in bilateral landing tasks. Future research should look to establish the variability for asymmetries in athletes regularly performing bilateral landing tasks as part of their competitive sport and training. 


\section{Louis Howe et al/2018}

The findings presented in this investigation should not be used for different landing tasks of a similar nature. As all of the kinetic variables measured in our investigation have been shown to differ between vertical $\mathrm{CMJ}$, forward jumps, single leg landings and bilateral drop-landings [12, 47], our findings should not be directly applied to other landing tasks. This has led to the functionality of the bilateral droplanding being questioned as it presents with differing task constraints from that of landing tasks that are preceded with a propulsive action (i.e. jumping) [47]. However, in contrast to screening landings from a CMJ, the bilateral drop-landing allows for practitioners to easily control the downward velocity at impact with the ground [48]. In this sense, the bilateral drop-landing may allow for an athlete's landing mechanics to be screened in a controlled manner, whilst being able to identify potential risk factors for injury. Although it has not currently been shown that reducing modifiable risk factors for injury within the bilateral drop-landing may alter landing mechanics in other landing tasks, it is likely that the skills required are transferable.

\section{Practical applications}

With such high force demands being placed on an athlete's musculoskeletal system during bilateral landing tasks, injury risk is clearly a primary consideration for practitioners. With portable force platforms being affordable and accessible to coaches, the reliability of kinetic variables related to landing performance has been presented in this study. Our investigation showed that peak VGRF, time to peak vGRF, loading rate and asymmetry in peak vGRF possessed relative reliability values ranging from large to near perfect. However, the signal to noise values suggest that drop height will likely influence the variability observed in force-time measures from bilateral landing. Specifically, CV\% measured for both legs and for a single-limb during bilateral droplandings decreased for peak vGRF, time to peak vGRF, and loading rate with greater drop heights. This is an important consideration for practitioners, [7] with measurement error for kinetic variables being influenced by drop height in relation to an individual's CMJ performance. In instances where the performance of a landing task is assessed without an appreciation for drop height relative to an individual's maximum CMJ height, there is potential for error in interpreting force-time variables between athletes. Based on our data, we suggest drop heights of $150 \%$ of an individuals maximum CMJ height be used so to provide greater reliability for assessing drop-landing kinetics.

\section{References}

[1] J.S. Dufek and B.T. Bates, Biomechanical factors associated with injury during landing in jump sports, Sports Medicine, 12 (1991) 326-337.

[2] J. Yanci, and J. Camara, Bilateral and unilateral vertical ground reaction forces and leg asymmetries in soccer players, Biology of Sport, 33 (2016) 179-183.

[3] G.H. Wadley and J.P. Albright, Women's intercollegiate gymnastics: injury patterns and "permanent" medical disability, American Journal of Sports Medicine, 21 (1993) 314320.

[4] N.A. Bates, K.R. Ford, G.D. Myer and T.E. Hewett, Impact differences in ground reaction force and center of mass between the first and second landing phases of a drop vertical jump and their implications for injury risk assessment, Journal of Biomechanics, 46 (2013) 1237-1241.

[5] D.R. Ortega, E.C.R. Bíes, and F.J.B.de la Rosa Analysis of the vertical ground reaction forces and temporal factors in the landing phase of a countermovement jump, Journal of Sports Science and Medicine, 9 (2010) 282287.

[6] C.H. Yeow, P.V.S. Lee, and J.C.H. Goh, Nonlinear flexion relationships of the knee with the hip and ankle, and their relative postures during landing, Knee, 18 (2011) 323-328.

[7] C.M. Fong, J.T. Blackburn, M.F. Nocross, M. McGrath, and D.A. Padua, Ankle-dorsiflexion 
range of motion and landing biomechanics, [15 Journal of Athletic Training, 46 (2011) 5-10.

[8] T.E. Hewett, G.D. Myer, K.R. Ford, R.S. Heidt Jr, A.J. Colosimo, S.G. McLean, A.J. Van den Bogert, M.V. Paterno and P. Succop, Biomechanical measures of neuromuscular control and valgus loading of the knee predict anterior cruciate ligament injury risk in female athletes: a prospective study, The American Journal of Sports Medicine, 33 (2005) 492501.

[9] M.F. Norcross, J.T. Blackburn, B.M. Goerger and DA. Padua, The association between lower extremity energy absorption and biomechanical factors related to anterior cruciate ligament injury, Clinical Biomechanics, 25 (2010) 1031-1036.

[10] R.W. Bisseling, A.L. Hof, S.W. Bredeweg, J. Zwerver and T. Mulder, Are the take-off and landing phase dynamics of the volleyball spike jump related to patellar tendinopathy?, British Journal of Sports Medicine, 42 (2008) 483-489.

[11] S.P. Bird and W.J. Markwick, Musculoskeletal screening and functional testing: considerations for basketball athletes, International Journal of Sports Physical Therapy, 11 (2016) 784-802.

[12] M.A.D. Britto, P.S. Franco, E. Pappas, and F.P. Carpes, Kinetic asymmetries between forward and drop jump landing tasks, The Revista Brasileira de Cineantropometria e Desempenho Humano, 17 (2015) 661-671.

[13] M.K. Rowley and J.G. Richards, Increasing plantar flexion angle during landing reduces vertical ground reaction forces, loading rates and the hip's contribution to support moment within participants, Journal of Sports Sciences, 33 (2015) 1922-1931.

[14] P.K. Schot, B.T. Bates and J.S. Dufek, Bilateral performance symmetry during drop landing: a kinetic analysis, Medicine \& Science in Sports \& Exercise, 26 (1994) 1153-1159.
[15] E.L. Radin, K.H. Yang, C. Riegger, V.L. Kish and J.J. O'Connor, Relationship between lower limb dynamics and knee joint pain, Journal of Orthopaedic Research, 9 (1991) 398-405.

[16] G. Atkinson and A.M. Nevill, Statistical methods for assessing measurement error (reliability) in variables relevant to sports medicine, Sports Medicine, 26 (1998) 217238.

[17] S. Cormack, R. Newton, M. McGuigan and T. Doyle, Reliability of measures obtained during single and repeated countermovement jumps, International Journal of Sports Physiology and Performance, 3 (2008) 131144.

[18] N. Hori, R.U. Newton, N. Kawamori, M.R. McGuigan, W.J. Kraemer and K. Nosaka, Reliability of performance measurements derived from ground reaction force data during countermovement jump and the influence of sampling frequency, The Journal of Strength and Conditioning Research, 23 (2009) 874-882.

[19] G. Markovic, D. Dizdar, I. Jukic and M. Cardinale, Reliability and factorial validity of squat and countermovement jump tests, The Journal of Strength and Conditioning Research,18 (2004) 551-555.

[20] F. Slinde, C. Suber, L.Suber, C.E. Edwén and U. Svantesson, Test-retest reliability of three different countermovement jumping tests, The Journal of Strength and Conditioning Research, 22 (2008) 640-644.

[21] C.R. James, J.A. Herman, J.S. Dufek and B.T. Bates, Number of trials necessary to achieve performance stability of selected ground reaction force variables during landing. Journal of Sports Science and Medicine, 6 (2007) 126-134.

[22] T.C. Mauntel, R.L. Begalle, T.R. Cram, B.S. Frank, C.J. Hirth, T. Blackburn and D.A. Padua, The effects of lower extremity muscle activation and passive range of motion on single-leg squat performance, The Journal of 


\section{Louis Howe et al/2018}

Strength and Conditioning Research, 27 (2013) 1813-1823.

[23] N. Benjanuvatra, B.S. Lay, J.A.Alderson and B.A. Blanksby, Comparison of ground reaction force asymmetry in one-and twolegged countermovement jumps, The Journal of Strength and Conditioning Research, 27 (2013) 2700-2707.

[24] G.L. Moir, Three different methods of calculating vertical jump height from force platform data in men and women, Measurement in Physical Education and Exercise Science, 12 (2008) 207-218.

[25] C.R. James, B.T. Bates and J.S. Dufek, Classification and comparison of biomechanical response strategies for accommodating landing impact, Journal of Applied Biomechanics, 19 (2003) 106-118.

[26] C.E. Milner, J.T. Fairbrother, A. Srivatsan and S. Zhang, Simple verbal instruction improves knee biomechanics during landing in female athletes, Knee, 19 (2012) 399-403.

[27] B.D. Roewer, K.R. Ford, G.D. Myer, and T.E. Hewett, The 'impact' of force filtering cut-off frequency on the peak knee abduction moment during landing: artefact or 'artifiction'?, British Journal of Sports Medicine, 48 (2014) 464-468.

[28] M.C. Hoch, K.E. Farwell, S.L. Gaven and J.T. Weinhandl, Weight-bearing dorsiflexion range of motion and landing biomechanics in individuals with chronic ankle instability. Journal of Athletic Training, 50 (2015) 833839.

[29] M.V. Paterno, K.R. Ford, G.D. Myer, R. Heyl and T.E. Hewett, Limb asymmetries in landing and jumping 2 years following anterior cruciate ligament reconstruction, Clinical Journal of Sport Medicine, 17 (2007) 258-262.

[30] M.J. Jordan, P. Aagaard and W. Herzog, Lower limb asymmetry in mechanical muscle function: a comparison between ski racers with and without ACL reconstruction,
Scandinavian Journal of Medicine \& Science in Sports, 25 (2015) 301-309.

[31] Field, A. Discovering statistics using IBM SPSS statistics. London: SAGE Publications Ltd, 2013.

[32] Hopkins W. A Scale of Magnitudes for Effect Statistics

[Internet] http://www.sportsci.org/resource/stats/index.h tml. A New View of Statistics. Internet Society for Sport Science, 2002.

[33] B.L. Riemann and M.R. Lininger, Statistical primer for athletic trainers: the essentials of understanding measures of reliability and minimal important change, Journal of Athletic Training, 53 (2018) 98-103.

[34] Hopkins W. Spreadsheets for Analysis of Validity and Reliability (Excel spreadsheet) [Internet]

http://www.sportsci.org/resource/stats/index.h tml. Internet Society for Sports Science, 2015.

[35] M.S. Walsh, K.R. Ford, K.J. Bangen, G.D. Myer and T.E. Hewett, The validation of a portable force plate for measuring force-time data during jumping and landing tasks, The Journal of Strength and Conditioning Research, 20 (2006) 730-734.

[36] M. Stokes, Reliability and repeatability of methods for measuring muscle in physiotherapy, Physiotherapy: Theory and Practice, 1 (1985) 71-6.

[37] V. Vu, A. Walker, N. Ball and W. Spratford, Ankle restrictive firefighting boots alter the lumbar biomechanics during landing tasks, Applied Ergonomics, 65 (2017) 123-129.

[38] S.N. Zhang, B.T. Bates and J.S. Dufek, Contributions of lower extremity joints to energy dissipation during landings, Medicine \& Science in Sports \& Exercise, 32 (2000) 812-819.

[39] J.G. Seegmiller and ST. McCaw, Ground reaction forces among gymnasts and recreational athletes in drop landings, Journal of Athletic Training, 38 (2003) 311-314.

[40] A.R. Mason-Mackay, C. Whatman and D. Reid, The effect of reduced ankle dorsiflexion 
on lower extremity mechanics during landing: A systematic review, Journal of Science and Medicine in Sport 20 (2017) 451-458.

[41] CR James, B.W. Scheuermann and M.P. Smith, Effects of two neuromuscular fatigue protocols on landing performance, Journal of Electromyography and Kinesiology, 20 (2010) 667-675.

[42] A.D. Nordin and J.S. Dufek, Lower extremity variability changes with drop-landing height manipulations, Research in Sports Medicine, 25 (2017) 144-155.

[43] C. Bishop, A. Turner and P. Read, Effects of inter-limb asymmetries on physical and sports performance: a systematic review, Journal of Sports Sciences, 36 (2018) 1135-1144.

[44] E. Pappas and F.P. Carpes, Lower extremity kinematic asymmetry in male and female athletes performing jump-landing tasks, Journal of Science and Medicine in Sport, 15 (2012) 87-92.

[45] L. Seifert, C. Button and K. Davids, Key properties of expert movement systems in sport, Sports Medicine, 43 (2013) 167-178.

[46] H.J.S. Shih, D.N. Jarvis, P Mikkelsen, and K. Kulig, Interlimb force coordination in ratecontrolled dance specific bipedal jumps: comparison between experts and novices, Journal of Applied Biomechanics, 29 (2018) $1-23$.

[47] J.R. Harry, J.F. Silvernail, J.A. Mercer and J.S. Dufek, Bilateral comparison of vertical jump landings and step-off landings from equal heights, The Journal of Strength and Conditioning Research, 32 (2018) 1937-1947.

[48] M. Afifi and R.N. Hinrichs, A mechanics comparison between landing from a countermovement jump and landing from stepping off a box, Journal of Applied Biomechanics, 28 (2012) 1-9. 


\section{Louis Howe et al/2018}

\section{Acknowledgement}

The authors did not receive any funding or grant support for the study.

Competing Interests

The authors declare that they have no competing interests.

\section{About The License}

\section{(c) (1)}

The text of this article is licensed under a Creative Commons

Attribution 4.0 International (CC BY 4.0) Attribution 4.0 International License 